\title{
Solar flares as proxy for the young Sun: satellite observed thermosphere response to an X17.2 flare of Earth's upper atmosphere
}

\author{
S. Krauss ${ }^{1}$, B. Fichtinger ${ }^{2}$, H. Lammer ${ }^{3}$, W. Hausleitner ${ }^{1}$, Yu. N. Kulikov ${ }^{4}$, I. Ribas ${ }^{5}$, V. I. Shematovich ${ }^{6}$, D. Bisikalo ${ }^{6}$, \\ H. I. M. Lichtenegger ${ }^{7}$, T. V. Zaqarashvili ${ }^{3}$, M. L. Khodachenko ${ }^{3}$, and A. Hanslmeier ${ }^{2}$ \\ ${ }^{1}$ Space Research Institute, Dept. of Satellite Geodesy, Austrian Academy of Sciences, Schmiedlstr. 6, 8042 Graz, Austria \\ ${ }^{2}$ University of Graz, Institute of Physics, Dept. of Astrophysics, Geophysics and Meteorology, Universitätsplatz 5/II, \\ 8010 Graz, Austria \\ ${ }^{3}$ Space Research Institute, Dept. of Extraterrestrial Physics, Austrian Academy of Sciences, Schmiedlstr. 6, 8042 Graz, \\ Austria \\ ${ }^{4}$ Polar Geophysical Institute, Russian Academy of Sciences, Khalturina Str. 15, 183010 Murmansk, Russian Federation \\ ${ }^{5}$ Institut d'Estudis Espacials de Catalunya/CSIC, Campus UAB, Facultat de Ciències, Torre C5-parell-2a planta, 08193 \\ Bellaterra, Spain \\ ${ }^{6}$ Institute of Astronomy, Russian Academy of Sciences, 48 Pyatnitskaya Str., 119017 Moscow, Russian Federation \\ ${ }^{7}$ Space Research Institute, Dept. Experimental Space Research, Austrian Academy of Sciences, Schmiedlstr. 6, 8042 Graz, \\ Austria
}

Correspondence to: S. Krauss (sandro.krauss@oeaw.ac.at)

Received: 6 February 2012 - Revised: 13 July 2012 - Accepted: 18 July 2012 - Published: 9 August 2012

\begin{abstract}
We analyzed the measured thermospheric response of an extreme solar X17.2 flare that irradiated the Earth's upper atmosphere during the so-called Halloween events in late October/early November 2003. We suggest that such events can serve as proxies for the intense electromagnetic and corpuscular radiation environment of the Sun or other stars during their early phases of evolution. We applied and compared empirical thermosphere models with satellite drag measurements from the GRACE satellites and found that the Jacchia-Bowman 2008 model can reproduce the drag measurements very well during undisturbed solar conditions but gets worse during extreme solar events. By analyzing the peak of the X17.2 flare spectra and comparing it with spectra of young solar proxies, our results indicate that the peak flare radiation flux corresponds to a hypothetical Sun-like star or the Sun at the age of approximately $2.3 \mathrm{Gyr}$. This implies that the peak extreme ultraviolet (EUV) radiation is enhanced by a factor of about 2.5 times compared to today's Sun. On the assumption that the Sun emitted an EUV flux of that magnitude and by modifying the activity indices in the JacchiaBowman 2008 model, we obtain an average exobase temperature of $1950 \mathrm{~K}$, which corresponds with previous theoreti-
\end{abstract}

cal studies related to thermospheric heating and expansion caused by the solar EUV flux.

Keywords. Atmospheric composition and structure (Evolution of the atmosphere; Pressure, density, and temperature) Solar physics, astrophysics, and astronomy (Flares and mass ejections)

\section{Introduction}

The escape and evolution of planetary atmospheres is strongly related to the evolution of the soft X-ray (SXR) and extreme ultraviolet (EUV) radiation and the particle environment of the planet's host star. From multi-wavelength observations and studies of solar analogue stars, so-called solar proxies, one can conclude that the solar flux integrated over $0.1-120 \mathrm{~nm}$ was higher by a factor of $\sim 6$ about $3.5 \mathrm{Gyr}$ ago, and larger than 20 times that of the present Sun about $4.2 \mathrm{Gyr}$ ago (Zahnle et al., 1982; Güdel et al., 1997; Guinan et al., 2003; Ribas et al., 2005; Güdel, 2007). 


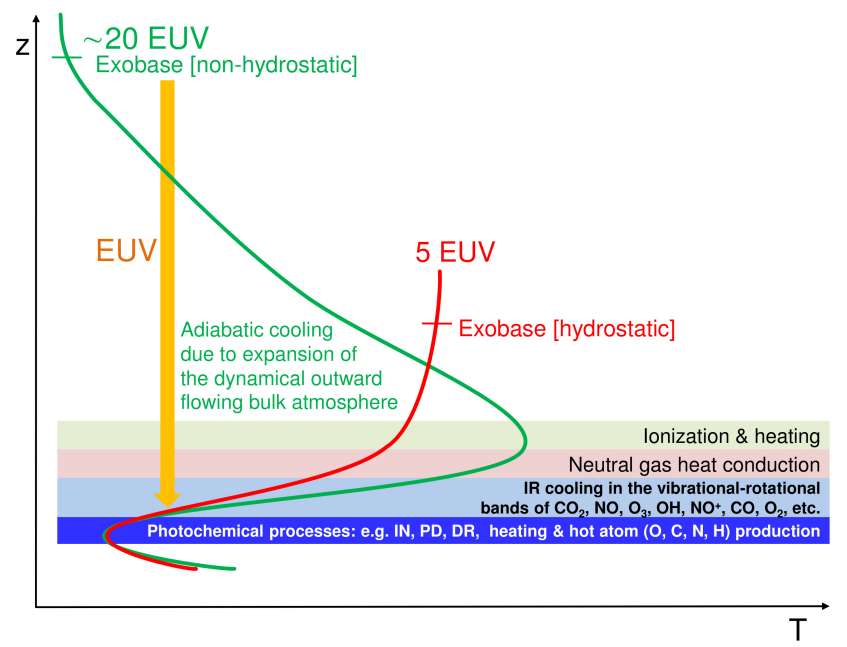

Fig. 1. Illustration of the thermospheric temperature response to high solar/stellar EUV flux. Enhanced ionization (IN) and photochemical processes like photo-dissociation (PD) and dissociative recombination (DR) eventually lead to heating and subsequent expansion of the upper atmosphere and to the production of suprathermal atoms which may influence the energy balance in the thermosphere. Depending on atmospheric species and energy deposition, upper atmospheres can switch from hydrostatic (Kulikov et al., 2007) to hydrodynamic regimes (Tian et al., 2008a,b).

The region of the Earth's atmosphere where the main part of the EUV radiation is absorbed and a substantial fraction of its energy is transformed into heat extends from about 90 to $500 \mathrm{~km}$. In order to understand the possible impact of the stronger solar EUV radiation on a terrestrial thermosphere and its enhanced erosion due to atmospheric loss processes during the early stages of the solar evolution, Kulikov et al. (2007) developed a hydrostatic, diffusive equilibrium and thermal balance model. They assumed present time chemical composition and included atmospheric heating by photoionization and photodissociation processes, heating in exothermic chemical reactions, cooling by infrared- (IR) radiating molecules such as $\mathrm{CO}_{2}, \mathrm{NO}, \mathrm{OH}$, and heat transfer by thermal and eddy conduction. The model simulations resulted in a heated and extended terrestrial thermosphere, a condition which should have lead to a diffusion-limited atomic hydrogen blow-off and high Jeans escape rates for heavier species during the first billion years after the Sun arrived at the zero-age main sequence (ZAMS). However, Tian et al. $(2008 \mathrm{a}, \mathrm{b})$ questioned that the thermosphere with its present chemical composition would have remained in hydrostatic equilibrium under extremely high temperatures of more than $10000 \mathrm{~K}$ during the first several hundred million years of the solar evolution. Tian et al. (2008a,b) developed a multicomponent hydrodynamic thermosphere model to probe the Earth's thermosphere under extreme solar EUV conditions. They found that in the case that the EUV input is higher than $\sim 5$ times the present EUV flux, the hydrostatic equi- librium regime transits to a hydrodynamic regime, resulting in reduced exobase temperatures and a lower Jeans loss rate of the atmosphere compared with the hydrostatic assumption (Fig. 1). Nevertheless, the exosphere could still expand efficiently even beyond the protecting magnetopause and the early Earth, with its assumed present time atmospheric composition, would have been in danger of losing all of its initial $\mathrm{N}_{2}$ inventory (Lichtenegger et al., 2010).

According to these results, the early Earth would have lost its $\sim 0.8$ bar $\mathrm{N}_{2}$ atmosphere (Encrenaz, 2001) within a few million years due to non-thermal loss of $\mathrm{N}^{+}$ions picked up by the solar wind. The findings also indicate that a higher amount of IR-cooling gas, such as $\mathrm{CO}_{2}$, in the early Earth atmosphere might have protected the thermosphere from extreme heating, expansion and finally from complete destruction. These recent results, which are based mainly on theoretical methods, may have tremendous implications for studies related to the early Earth or in general for $\mathrm{N}_{2}$-rich atmospheres of Earth-type planets orbiting within the habitable zones of EUV-active M-stars (Scalo et al., 2007; Lammer et al., 2009). Therefore, a validation of the theoretical model results by observations from low Earth orbiting (LEO) satellites, such as GRACE (Gravity Recovery and Climate Experiment), or observation-based empirical models, like the NRLMSISE-00 (MSIS00, Picone et al., 2003) or the Jacchia-Bowman 2008 model (JB08, Bowman et al., 2008), is highly desirable. Since GRACE is orbiting the Earth below the exobase at $\sim 500 \mathrm{~km}$ altitude, it can be used as a diagnostic tool for retrieving thermospheric density and its variations during extreme solar events, which can be considered as proxies for periods when the Sun was younger and more active.

The effects of extremely powerful solar flares and energetic particle events on a planetary upper thermosphere and ionosphere have also been observed on Venus and Mars with spacecraft (S/C) such as Mars Global Surveyor (MGS), and Mars and Venus Express (MEX, VEX) (e.g. Leblanc et al., 2002; Crider et al., 2005; Espley et al., 2005; Futaana et al., 2008).

In this context, Crider et al. (2005) studied the effects of an encounter of a massive cloud of solar plasma, which was generated by a coronal mass ejection (CME) associated with the X17.2 solar flare on 28 October 2003, with the martian space environment. The electron spectra measured by the electron reflectometer instrument (ER) onboard the MGS indicated that solar plasma penetrated into the martian oxygen corona as deep as $\sim 400 \mathrm{~km}$ altitude during the extreme event, whereas prior to that the bottom of the magnetosheath was typically observed at around $900-1200 \mathrm{~km}$. Thus, during the extreme storm, high energy solar wind protons and electrons interact more efficiently with atmospheric neutrals via charge exchange and electron impact ionization compared to quiet times, resulting in an increase of the atmospheric loss rates. 
Also based on MGS/ER observations on Mars, Espley et al. (2005) analyzed powerful plasma oscillations and their possible connection with enhanced atmospheric loss during the extreme solar storm in October 2003. The authors suggested that the occurrence of strong plasma oscillation activity, across an extended magnetotail region of the planet, might have lead to substantially increased atmospheric loss due to enhanced efficiency of the ion pickup mechanism.

Most recently, Futaana et al. (2008) analyzed a large enhancement in count rates of heavy ions, which has been recorded by ASPERA instruments aboard the MEX and VEX S/C during a strong X9.0 solar flare in December 2006. This first observation of a strong atmospheric ion flux increase associated with the solar flare is a clear indication that in the early history of the solar system extreme solar activity events could strongly affect atmospheric loss processes and the evolution of terrestrial planets' atmospheres.

This present work is an interdisciplinary study that focuses on the response of the Earth's thermosphere to an extreme solar flare which occurred during the so-called "Halloween period" in 2003. We investigate the flare-related enhanced EUV radiation in combination with the spectra of the solar analogue stars having younger age. In Sect. 2 we describe the X17.2 flare that is investigated in our study. We analyze the spectra of the flare in the EUV range by using the correlated data from the Coronal Diagnostic Spectrometer (CDS) aboard the Solar and Heliospheric Observatory (SOHO) and the Solar EUV Experiment (SEE) aboard the Thermosphere Ionosphere Mesosphere Energetics and Dynamics (TIMED) satellite. The analyzed EUV enhancement due to the flare is compared with spectra of solar proxies, and the flare-related EUV activity is determined as a proxy for the young Sun. In Sect. 3 we discuss in-situ observations based on accelerometer drag measurements aboard the GRACE spacecraft $(\mathrm{S} / \mathrm{C})$. We describe how we analyzed and calibrated the satellite drag data and deduced the ambient neutral atmosphere densities before, during, and after the X17.2 flare event. Neutral densities calculated from the resulting dynamic drag force coefficient are compared with densities using a constant drag coefficient. In Sect. 4 we compare neutral densities from GRACE observations with those supplied by the empirical thermosphere models MSIS00 and JB08 that use daily averaged solar activity indices. In the last section we re-evaluate the empirical thermosphere model JB08 using pseudo solar activity indices, which are related to the activity maximum of the flare event. The resulting thermospheric densities and exobase temperatures are compared with results from theoretical models. Finally, we discuss the implications of our results for Earth-type $\mathrm{N}_{2}$ atmospheres which are exposed to higher solar or stellar EUV fluxes.

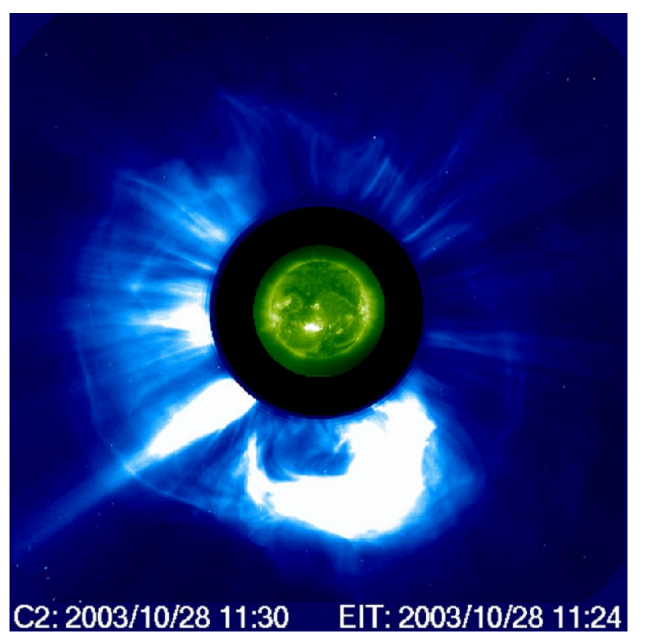

Fig. 2. Image of the X17.2 flare on 28 October 2003 recorded with SOHO/EIT (Extreme ultraviolet Imaging Telescope) and the associated halo CME recorded with SOHO/LASCO (Large Angle and Spectrometric Coronagraph). Pictures: NASA/ESA.

\section{Analysis of the X17.2 Halloween flare event and com- parison with young solar proxies}

During the spectacular solar activity period, called "Halloween period" in late October and early November 2003, several X-class flares, more than 40 coronal mass ejections (CMEs), five solar energetic particle events, and two strong geomagnetic storms occurred. One of these extreme events was the X17.2 flare on 28 October. This flare is illustrated in Fig. 2 with the lightened region near the center of the disc.

The strong flare started at 09:51 UT, had its maximum peak at 11:10 UT and ended at 11:24 UT resulting in an exposure time of approximately $90 \mathrm{~min}^{1}$. It was associated with a very fast halo CME, located in the active region AR 10486 at S16E08. Through the impressive velocity, which was larger than $2200 \mathrm{~km} \mathrm{~s}^{-1}$ in interplanetary space, the CME was one of the fastest ever recorded. For example, the United States Geological Survey Boulder magnetometer ${ }^{2}$ measured a sudden impulse of $140 \mathrm{nT}$ at 06:13 UT on 29 October, equivalent to a transit time of merely $19 \mathrm{~h}$. The huge solar eruption caused electrical blackouts in southern Sweden and damaged several transformers in South Africa. The powerful EUV radiation of the solar flare and the subsequent strong particle emissions of relativistic protons, gradual and impulsive electrons due to the CME had immense consequences for the upper atmosphere of the Earth. The SOHO/CDS spectrometer, which provides information about the characteristics of the solar coronal plasma such as density, temperature, flow velocity, fluxes, abundances, etc. on spatial, temporal and spectral scales is used to obtain the intensity flux of the flare in EUV. The CDS consists of a Wolter-Schwarzschild type 2

\footnotetext{
${ }^{1}$ http://vso.nso.edu/cgi/catalogui

${ }^{2}$ http://www.swpc.noaa.gov/rt_plots/bou_12h.html
} 


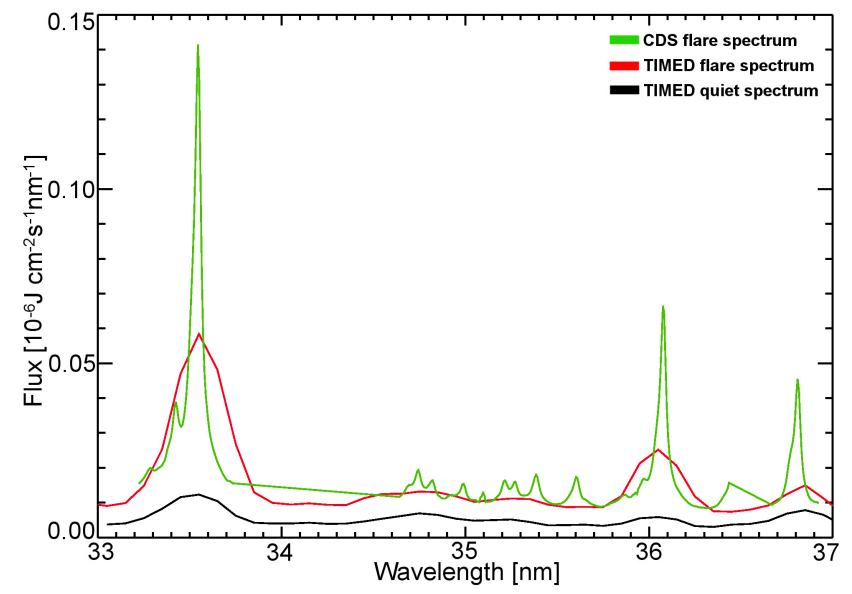

Fig. 3. Flare spectrum in nm during the maximum of the X17.2 flare on 28 October 2003 at 11:02 UT shown as green (SOHO/CDS) with a resolution of $0.03 \mathrm{~nm}$ and red (TIMED/SEE) at 11:17 UT with a resolution of $0.4 \mathrm{~nm}$. The black spectrum illustrates the quiet Sun during moderate solar activity with the intensity flux being 2.5 times lower than during the flare.

telescope, a set of slits, a scan mirror and two spectrometers, called the normal incidence spectrometer (NIS) and the grazing incidence spectrometer (GIS). After applying corrections and calibration routines, the EUV spectrum at the flare maximum can be specified. Because of a gap between $37-52 \mathrm{~nm}$, data of a second satellite, TIMED, are additionally used for the analysis. The TIMED/SEE instrument determines the irradiance of the highly variable solar EUV radiation. The satellite is able to observe the Sun approximately 12 to 15 times per day, and each observation lasts about three minutes. Additionally, daily averages of solar irradiances are provided. Since TIMED covers a wavelength range of $27-$ $195 \mathrm{~nm}$, larger than CDS, it is an important instrument for investigating the solar flare. For our short-term flare studies, we use Level 2A data from the SEE EUV Grating Spectrograph (EGS), which contain the three minute irradiance observations.

Based on these TIMED/SEE data, we calculated the integral of the intensity flux over the EUV range (25-92 nm) during the flare maximum and for the quiet Sun during moderate solar activity. A comparison showed that the intensity flux of the flare maximum is $\sim 2.5$ times higher and therefore exceeds the flux at high solar activity. Figure 3 illustrates the intensity flux per nanometer during the maximum of the flare.

To reconstruct the history of the evolution of the young Sun, the flare spectrum is compared to spectra of solar analogs of younger ages. The "Sun in Time" program (Dorren and Guinan, 1994; Güdel, 2007; Guinan et al., 2003) was established to study a series of solar-like stars of different ages back to the ZAMS in multi-wavelength observations (e.g. Ribas et al., 2005; Güdel, 2007). Power laws of Ribas et al. (2005) couple the intensity flux in a specific wavelength

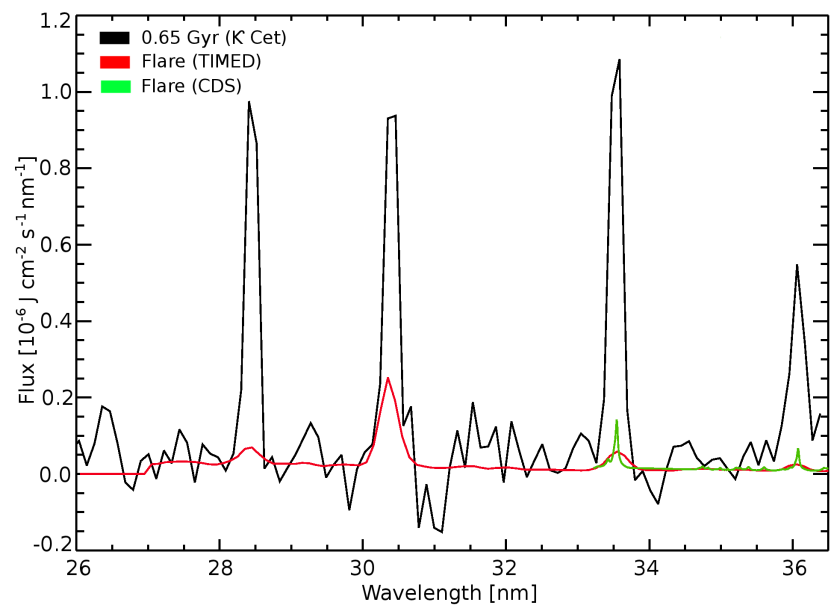

Fig. 4. Comparison of the EUV spectra of the X17.2 flare representing the $2.3 \mathrm{Gyr}$ old Sun and the younger $0.65 \mathrm{Gyr}$ old solar proxy $\kappa^{1}$ Cet that shows higher emission than the flare event.

region with the stellar age. In the extreme ultraviolet (36$92 \mathrm{~nm}$ ) the power law can be written as:

$F_{\mathrm{EUV}}=4.56 \tau^{-1.0}$,

where $F_{\mathrm{EUV}}$ is the intensity flux given in $10^{-7} \mathrm{~J} \mathrm{~cm}^{-2} \mathrm{~s}^{-1}$, and $\tau$ is the age in Gyr. Evaluating Eq. (1) shows that the intensity of the Halloween flare corresponds to a Sun-like star at the age of about $2.3 \mathrm{Gyr}$. Table 1 presents the integrated fluxes of several solar proxies of different ages in various wavelength ranges including several emission lines, where the integrated fluxes of the solar-like star related to the specific flare event at the age of $\sim 2.3 \mathrm{Gyr}$ determined with TIMED and CDS correspond very well to the results published by Ribas et al. (2005).

Figure 4 shows, as an example, a comparison based on the EUV spectrum of the solar flare during its maximum and the spectrum of the $0.65 \mathrm{Gyr}$ old solar proxy $\kappa^{1}$ Cet (Table 1 , col. 4). It can be recognized that the intensity flux of the young solar proxy is $\sim 7$ times higher compared to the intensity of the analyzed flare.

\section{Solar activity related in-situ measurements in the Earth's upper atmosphere}

Since 2002 the GRACE twin satellite mission (Tapley et al., 2004) has been orbiting at the upper part of the thermosphere. The S/C fly about $220 \mathrm{~km}$ apart in a near-circular polar orbit. The initial altitude was $505 \mathrm{~km}$, a height level which nearly coincides with the upper boundary of the exobase. Among a number of instruments, the satellites carry the SuperSTAR accelerometer sensors built by the Office National d'Etudes et de Recherches Aerospatials (ONERA). By analyzing the measured data, it is possible to obtain information concerning the change of the neutral density at a specific altitude, 
Table 1. Integrated fluxes of different solar analogues (Ribas et al., 2005) and a corresponding 2.3 Gyr old solar proxy determined from the $\mathrm{X} 17.2$ Halloween flare analysis in units of $10^{-7} \mathrm{~J} \mathrm{~cm}^{-2} \mathrm{~s}^{-1}$. Two wavelength ranges in the EUV, the FUV and eight strong emission lines are shown. The stars with ages 0.1 (EK Dra), 0.3 ( $\pi^{1} \mathrm{UMa}, \chi^{1}$ Ori), $0.65\left(\kappa^{1} \mathrm{Cet}\right)$ and $1.6 \mathrm{Gyr}(\beta \mathrm{Com})$ represent well studied solar analogues with younger age (Ribas et al., 2005), and the 4.56-Gyr-old one represents the present Sun.

\begin{tabular}{lcccccc}
\hline $\begin{array}{l}\text { Wavelength } \\
\text { range (nm) }\end{array}$ & $0.1 \mathrm{Gyr}$ & $0.3 \mathrm{Gyr}$ & $0.65 \mathrm{Gyr}$ & $1.6 \mathrm{Gyr}$ & $4.56 \mathrm{Gyr}$ & $\begin{array}{c}\text { X17.2 flare } \\
\text { eq. 2.3 Gyr }\end{array}$ \\
\hline $10-36$ & 187.2 & 69.4 & 22.7 & 7.7 & 2.05 & 2.7 \\
$36-92$ & 45.6 & 15.2 & 7.0 & 2.85 & 1.00 & 1.99 \\
$92-118$ & 18.1 & 8.38 & 2.90 & 1.70 & 0.74 & 1.64 \\
$33.5(\mathrm{Fe}$ XVI) & 36.6 & 9.7 & 2.6 & - & - & 0.11 \\
36.1 (Fe XVI) & 15.7 & 6.6 & 1.6 & - & 0.016 & 0.06 \\
$130.4(\mathrm{O} \mathrm{I})$ & 4.3 & 1.18 & 0.60 & 0.45 & 0.143 & 0.20 \\
133.5 (C II) & 4.7 & 1.52 & 0.95 & 0.36 & 0.18 & 0.28 \\
140.0 (Si IV) & 4.3 & 1.59 & 0.77 & 0.28 & 0.083 & 0.18 \\
155.0 (C IV) & 9.1 & 2.21 & 1.02 & 0.40 & 0.146 & 0.34 \\
164.0 (He II) & 6.0 & 0.99 & 0.56 & - & 0.04 & 0.23 \\
165.7 (C I) & 4.1 & 0.97 & 0.78 & 0.47 & 0.202 & 0.38 \\
\hline
\end{tabular}

Table 2. GRACE mission parameters and accelerometer specification. The satellite mass and mean altitude are given for the mission start in 2002 and the present time (1 January 2012).

\begin{tabular}{ll}
\hline Mission parameters & \\
\hline Eccentricity & $<0.005$ \\
Inclination & $89 \mathrm{deg}$ \\
Orbit period & $94 \mathrm{~min}$ \\
Initial/present mass & $487 \mathrm{~kg} / 471 \mathrm{~kg}$ \\
Cross sectional area & $0.96 \mathrm{~m}^{2}$ \\
Initial/present altitude & $505 \mathrm{~km} / 465 \mathrm{~km}$ \\
\hline SuperSTAR accelerometer specifications \\
\hline Measurement Bandwidth & $5 \times 10^{-5} \ldots 10^{-1} \mathrm{~Hz}$ \\
Measurement Range & $\pm 5 \times 10^{-5} \mathrm{~m} \mathrm{~s}^{-2}$ \\
Measurement Accuracy & $1 \times 10^{-10} \mathrm{~m} \mathrm{~s}^{-2}$ \\
\hline
\end{tabular}

latitude and time. Mission parameters as well as some accelerometer specifications are listed in Table 2.

The accelerometer is rigidly mounted at the center of the satellite mass and consists of a charged proof mass sitting in a cage that maintains an electrostatic field. The voltages, measured by a feedback loop to keep the proof mass at rest, are converted to accelerations and provided to the scientific community via the Information System and Data Center (ISDC) of the Geoforschungszentrum Potsdam. The raw data from the accelerometer contain information on linear and angular accelerations at a $1 \mathrm{~Hz}$ sampling rate. Quaternions, which are necessary for the transformation between various coordinate systems, are determined by us following Wu et al. (2006). Since the raw acceleration measurements $a_{\text {raw }}$ are highly influenced by instrumental biases, a data calibration has to be performed according to Bruinsma et al. (2007).

$a_{\text {cal }}=\left(a_{\text {raw }}-\right.$ bias $) \cdot$ scale.
Bias and scale factors, provided by the Centre National d'Études Spatiales (CNES), are estimated by processing GPS and accelerometer data in daily arcs, using accurate gravity field and ocean tide models.

In the present study we concentrate on the two most dominating forces acting on the satellite along the in-track axis, i.e. atmospheric drag and solar radiation pressure. Therefore, the above calibrated values must be reduced by the disturbing acceleration originating from the solar radiation to finally yield the contribution of the atmospheric drag only. The acceleration due to solar radiation pressure can be computed following Montenbruck et al. (2001) or Sutton et al. (2007):

$$
\begin{aligned}
a_{\mathrm{srp}}= & \sum_{i=1}^{9} \frac{S A_{\mathrm{i}} \cos \left(\phi_{\mathrm{inc}, \mathrm{i}}\right)}{m c} \\
& {\left[2\left(\frac{c_{\mathrm{rd}, \mathrm{i}}}{3}+c_{\mathrm{rs}, \mathrm{i}} \cos \left(\phi_{\mathrm{inc}, \mathrm{i}}\right)\right) \boldsymbol{n}_{\mathrm{i}}+\left(1+c_{\mathrm{rs}, \mathrm{i}}\right) \boldsymbol{s}_{\mathrm{i}}\right] . }
\end{aligned}
$$

Here, $S$ denotes the solar flux constant $\left(1366 \mathrm{~W} \mathrm{~m}^{-2}\right), c$ the speed of light and $m$ the satellite mass. The specific plate area $A_{\mathrm{i}}$ as well as the coefficients for specular and diffuse reflectivity $c_{\mathrm{rs}}$ and $c_{\mathrm{rd}}$ are taken from a 9-plate macro model for the GRACE S/C (Bettadpur, 2007). To compute the angle between the plate normal and the direction towards the Sun $\phi_{\text {inc }}$, the position vector of the Sun relative to the S/C is computed at every epoch. In this context, the vector $\boldsymbol{n}_{\mathrm{i}}$ denotes the $i$-th unit plate normal and $s_{\mathrm{i}}$ the $i$-th unit satellite-sun vector. When summing up the individual contributions from each plate, one has to keep in mind that some plates might be shaded by others and therefore may not contribute to the solution. Furthermore, shadowing effects from the Earth have to be taken into account. During the Halloween period the calculated accelerations due to the solar radiation pressure acting in the along track axis are up to $\pm 2 \times 10^{-8} \mathrm{~m} \mathrm{~s}^{-2}$ and have to be subtracted from the total accelerations in this 


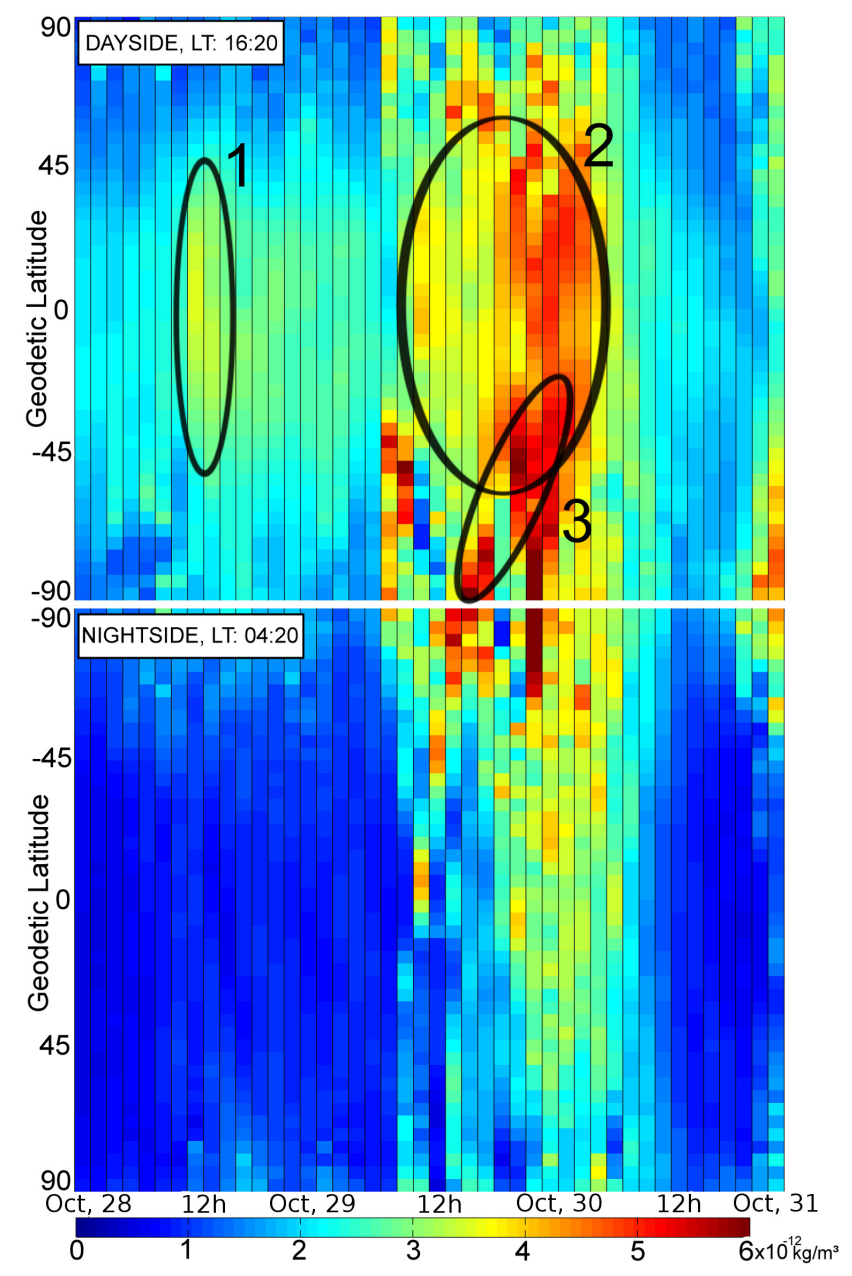

Fig. 5. Impact of the geomagnetic storm on the upper Earth atmosphere, in terms of normalized neutral densities $\rho_{490}$ determined from GRACE satellite accelerations during 28-30 October 2003 at the dayside (LT: 16:20) and at the nightside (LT: $\sim 04: 20$ ). The time interval between two adjacent vertical grid lines covers a single satellite revolution (94 min) and defines the time resolution of the plot. (1) corresponds to the measured density enhancement at the satellite orbit location caused by the X17.2 flare. (2) and (3) are density enhancements which originated a day after the flare exposure due to the accompanied CME and related high energy particle events.

direction. Given the reduced along track acceleration $a_{\mathrm{x}}$ of the S/C, the atmospheric drag force $F_{\mathrm{d}}$ acting on the satellite can be obtained following Moe and Moe (2005) by:

$F_{\mathrm{d}}=m a_{\mathrm{x}}=\frac{1}{2} \rho v_{\mathrm{inc}}^{2} C_{\mathrm{F}} A_{\text {ref }}$,

where $\rho$ denotes the total neutral density at the satellite orbit and $C_{\mathrm{F}}$ the drag force coefficient. Information concerning the actual satellite mass $m$, the velocity $v_{\text {inc }}$, and the reference area of the S/C surface $A_{\text {ref }}$ can be obtained from the GPS navigation data and a macro model of the S/C. The crucial factor is the force coefficient $C_{\mathrm{F}}$ and thus the gas-surface in- teraction. This coefficient is a highly complex and variable quantity and was therefore the object of countless studies during the last 50 years. On this account we make use of existing knowledge concerning this issue (see Appendix A).

According to our calculations, the resulting dynamic force coefficient has a sampling rate of one minute and varies between 2.8 and 3.5 during one satellite revolution. The main variation is caused by the elliptical satellite orbit, whereby the coefficient increases with decreasing altitude. In case of the GRACE S/C, the height difference from orbit eccentricity is approximately $50 \mathrm{~km}$. By inserting the variable $C_{\mathrm{F}}$ coefficient into Eq. (4), we can compute the neutral density with a resolution of one minute.

Figure 5 shows the resulting neutral density observed by GRACE, in a latitude-time plot during the Halloween period. Thereby, several irregularities appear, which can be attributed to the geomagnetic storm that occurred on 28 October at 11:10 UT. Due to the mentioned height differences along a satellite orbit, the densities are normalized to an average height level of $490 \mathrm{~km}$ (Bruinsma et al., 2006) by:

$\rho_{490}=\frac{\rho_{\mathrm{M} 490}}{\rho_{\mathrm{M}}} \rho$.

In order to receive the normalized density $\rho_{490}$ for GRACE, we have evaluated the empirical model JB08 at the S/C orbit and at a constant altitude of $490 \mathrm{~km}$, thus obtaining the density $\rho_{\mathrm{M}}$ and $\rho_{\mathrm{M} 490}$. These densities can then be used to normalize the actual neutral density $\rho$ observed by GRACE.

Shortly before 12:00 UT on 28 October, we observe the first sudden rise in neutral dayside densities of approximately $60 \%$ compared to the preceding density values at the equatorial and middle latitudes (Fig. 5, region 1). Even though the S/C entered the Earth shadow shortly before the eruption and remained on the nightside for $45 \mathrm{~min}$, this dayside density increase can still be related to the solar flare EUV photons that occurred at 11:10 UT.

The major impact appeared $19 \mathrm{~h}$ later, when the shock wave of the large CME disturbed the Earth's magnetic field. This lead to a significant increase of the drag and the accelerations and thus in the neutral density of more than $300 \%$ (Fig. 5, region 2) compared to the densities before the event. Unlike the density behaviour during the flare on 28 October, the density increased also on the nightside of the planet. During the night time the largest density enhancements were observed at high latitudes near the South Pole. One more important detail can be observed in Fig. 5 during 29 October on the dayside, namely a travelling atmospheric disturbance (TAD), which propagates from high latitudes towards the equator within a few satellite revolutions (Fig. 5, region 3). Such TADs are a response to sudden energy and momentum deposition in the high latitude thermosphere mostly in the form of Joule heating. For more information about the characteristics of TADs, the reader is referred to Bruinsma and Forbes $(2007,2008)$. 
In Fig. 6 satellite records of the geostationary GOES-10 satellite are illustrated. In the upper two panels, the course of the solar X-rays and the proton flux are given for the Halloween period in 2003.

A significant increase can be observed around noon on 28 October, when the X17.2 solar flare arises. Furthermore, we see an increase due to a X10.0 flare, which occurred at $\sim 20: 50$ on 29 October. Since the influences of this solar flare coincide with the impact of the CME, the events could not be separated in the analyses of the atmospheric densities. Finally, the bottom panel illustrates the measurements of the Earthward magnetic field. In accordance to the analysis of the mass densities, we can observe the perturbations originated by the CME on 29 October.

In the present study we limit our analysis to the effect of the enhanced EUV flux of the X17.2 flare on the upper atmosphere and its implications for the younger Sun. Thus, a comparison of in-situ measurements, empirical models and theoretical studies focusing on EUV heating of the Earth's early thermosphere is carried out. A similar analysis of the related energetic particle events on 29 October is beyond the scope of the present work but is planned to be performed in the future.

\section{Comparison of in-situ measurements with empirical thermosphere models}

Beside the density determination based on accelerometer measurements from GRACE, we also calculated total mass densities by using two empirical thermosphere models (MSIS00, JB08). In order to evaluate which model reproduces the calculated GRACE density variations resulting from various sources more precisely, we investigated the period of eight years (2003-2010).

The MSIS00 model is an extension of the previous MSIS86 and MSIS90 models. Contrary to these earlier models, it includes $\mathrm{O}_{2}$ data from the NASA Solar Maximum Mission and is applicable from ground to space. Referring to the main drivers of the upper atmosphere, the model uses the daily $10.7 \mathrm{~cm}$ solar radio flux $F_{10.7}$ as a proxy for the solar EUV and the three-hourly geomagnetic index $a_{\mathrm{p}}$ for the geomagnetic component of the space weather. The $a_{\mathrm{p}}$ index is deduced from the planetary index $K_{\mathrm{p}}$, which is determined from magnetic field measurements at several observatories around the world.

The JB08 model originates from the CIRA72 model (Jacchia, 1971). Unlike the MSIS00, which only uses the $F_{10.7}$ as a proxy for the solar EUV, this model includes three more solar indices $\left(S_{10.7}, M_{10.7}, Y_{10.7}\right)$ to map energy from specific solar irradiance sources to major thermospheric layers (Tobiska et al., 2008). This enabled the developers to include new semi-annual density variation equations using multiple 81-day average solar activity indices in order to take into account the variations in the semi-annual density cycle that re-

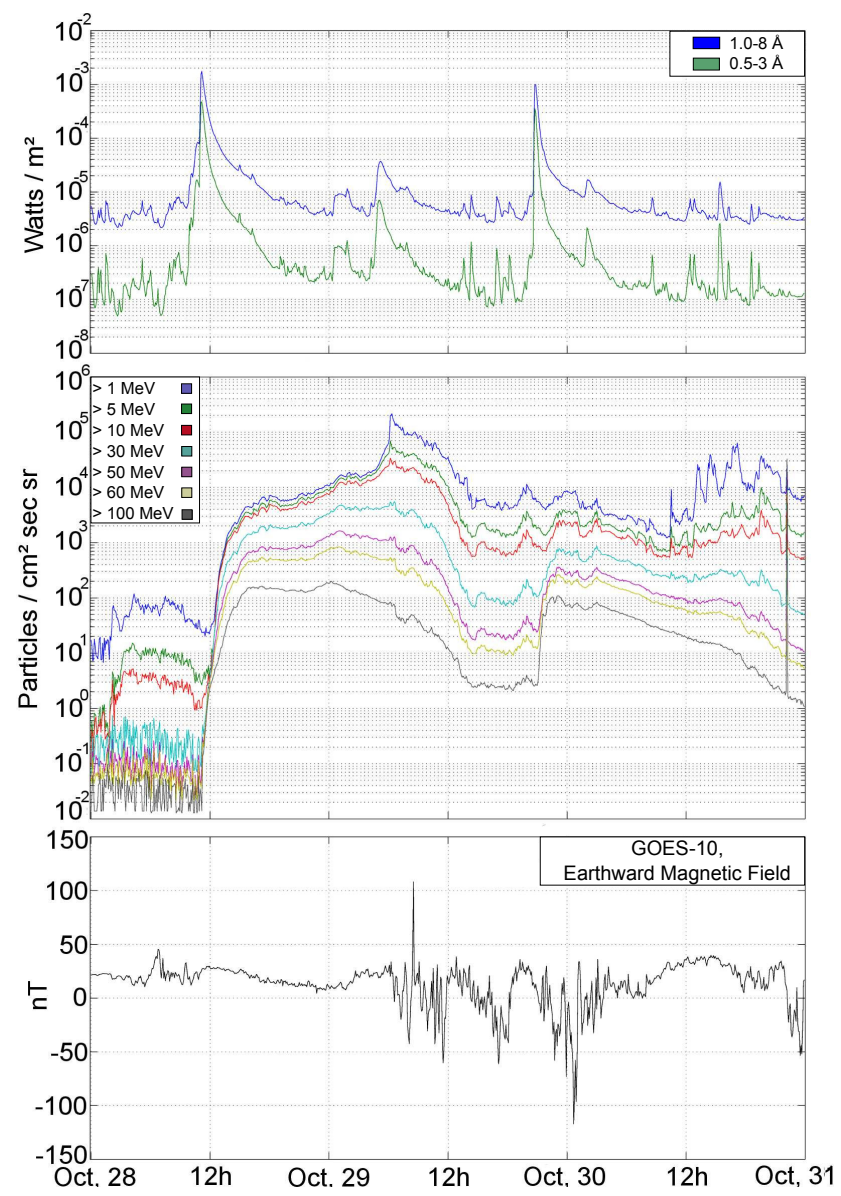

Fig. 6. Satellite measurements by the geostationary GOES-10 S/C during the Halloween period in 2003. The upper panel shows the course of the solar X-rays; in the middle panel the proton fluxes are illustrated. The bottom panel displays data records from the Earthward component of the magnetic field. All data records were obtained by the National Geophysical Data Center (NGDC).

sult from long-term EUV heating (Bowman et al., 2008). The $M_{10.7}$ index is thereby a proxy for the photospheric and lower chromospheric solar far ultraviolet (FUV) Schumann Runge Continuum emission near $160 \mathrm{~nm}$. The $S_{10.7}$ index is an activity indicator of the integrated $26-34 \mathrm{~nm}$ solar emission. Finally, the most recently implemented index $Y_{10.7}$ comprises competitive drivers to the mesosphere and lower thermosphere.

Additionally, exospheric temperature equations based on these EUV and FUV solar indices were introduced to represent the thermosphere EUV and FUV heating effects.

In contrast to the MSIS00 model, the JB08 uses two different geomagnetic indices depending on the present geomagnetic activity level. In principal, the model uses the threehourly $a_{\mathrm{p}}$ as an input parameter. However, in case of a geomagnetic storm $\left(a_{\mathrm{p}}>40\right)$ the model switches from the $a_{\mathrm{p}}$ to the Disturbance Storm Time index, $D_{\text {st }}$. This index is an indicator of the strength of the storm-time current in the inner 
Table 3. Percentage deviation of the total mass densities evaluated with the empirical models JB08 and MSIS00 from the reference solution based on GRACE acceleration measurements. Investigation period: 2003-2010.

\begin{tabular}{lccc}
\hline $\begin{array}{l}\text { Geomagnetic } \\
\text { Activity }\end{array}$ & Kp & $\begin{array}{c}\text { JB08 } \\
{[\%]}\end{array}$ & $\begin{array}{c}\text { MSIS00 } \\
{[\%]}\end{array}$ \\
\hline Quiet & $0-1$ & -1.2 & +16.6 \\
Unsettled & $2-3$ & +1.3 & +15.5 \\
Active & 4 & +2.4 & +16.4 \\
Minor Storms & 5 & +2.4 & +19.5 \\
Major Storms & 6 & +4.1 & +11.9 \\
Severe Storms & $7-9$ & +5.1 & +7.8 \\
\hline
\end{tabular}

magnetosphere with the advantage that it primarily responds to the magnetospheric variations, whereas the $a_{\mathrm{p}}$ responds mainly to the currents flowing in the ionosphere. Additionally, the higher time resolution of the $D_{\text {st }}$ index (one hour) makes it possible to segregate different storm phases (Bowman et al., 2008). Apart from total mass densities, both models also provide exospheric temperatures. Additionally, the MSIS00 model supplies densities of the most important atmosphere constituents.

We computed densities for the years 2003 to 2010, thus covering periods of high and low solar activity. In order to see how the empirical models perform during periods of different solar activity conditions, we divided the resulting densities into six classes based on the 3 -hourly $K_{\mathrm{p}}$ index, i.e. each sampling includes two satellite revolutions.

As can be seen from Table 3, especially the MSIS00 overestimates the measured GRACE densities, in general. However, for high activity the difference between measurement and the model decreases. In contrast to this, the JB08 shows a good agreement with the densities from the in-situ measurements, especially when the solar activity is low. By comparing the correlation coefficients between the models and the measurements, it can be seen that the best correlation is during low solar activity (JB08: 0.94, MSIS00: 0.93). With increasing solar activity the correlation coefficients decrease to 0.87 and 0.80 for the JB08 and the MSIS00 model, respectively.

Figure 7 shows the normalized dayside maximal atmospheric densities for each satellite revolution, inferred from the satellite-based accelerometer measurements and from the two empirical models during the Halloween period.

Particularly before the perturbations triggered by the energetic particles occur (Fig. 7, region 2), the results of the JB08 are in good agreement with the GRACE measurements, while the MSISO0 shows a significant overestimation. It is clearly evident that both models can not simulate the observed density response triggered by the solar flare (Fig. 7, region 1). However, it has to be noted that neither of the models are designed to simulate such short-term perturbation caused by solar radiation. For instance, the four solar indices

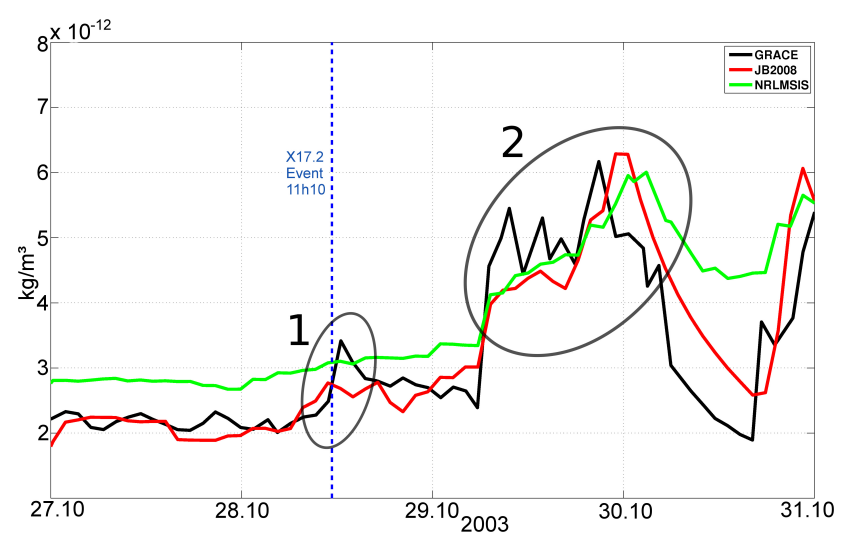

Fig. 7. Comparison of dayside atmospheric densities during the Halloween period based on GRACE accelerometer measurements (black line) and the empirical thermosphere models JB08 (red line) and MSISO0 (green line). The graphic shows the dayside maxima for each satellite revolution.

which are used as input parameters by the JB08 model are determined from satellite and ground-based observations with different cadences and are used as daily and 81-day averaged values. In order to model various physical and chemical processes in the Earth's atmosphere, each of these indices are applied with a specific lag time between one and five days. For example, the model uses the $S_{10.7}$ index with a delay of one day to be consistent with the average oxygen thermal conduction timescale in the thermosphere above $180 \mathrm{~km}$. Therefore, even if we calculate, e.g. high-resolution $S_{10.7}$ indices based on $15 \mathrm{~s}$ SOHO/SEM measurements during the flare event, it would not affect the model results.

In the case of the geomagnetic indices, a higher time resolution, ranging from one to three hours, is available. Hence, both empirical models show a better agreement with the insitu measurements when the shock wave of the CME disturbs the Earth's magnetic field (Fig. 7, region 2). However, we can observe that the density decay in time for both models is too slow, compared with the actual measurement. We conclude from our study that the JB08 model shows a distinctly better agreement with the in-situ measurements than the MSISO0 model. Consequently, we prefer the JB08 model for further comparisons in the following section.

\section{Comparison of theoretical and empirical thermo- sphere model results}

As already mentioned in the introduction, the multiwavelength observations of the X-ray, EUV, and UV radiation of solar type stars with different ages, performed during the past two decades by space telescopes, provided strong evidence that the young Sun was much more active than today (e.g. Ribas et al., 2005; Güdel, 2007). 
Table 4. Solar indices as daily average values for $F_{10.7}, S_{10.7}$, $M_{10.7}$ and $Y_{10.7}$ and 81-day average values for $F_{81 \mathrm{c}}, S_{81 \mathrm{c}}, M_{81 \mathrm{c}}$ and $Y_{81 \mathrm{c}}$ (column 2). Columns 3-5 show the calculated ratios $\Delta \mathrm{EUV} / \Delta I_{\mathrm{M}}$, the multiplication factors $k$, as well as the resulting pseudo indices, representing the maximum of the X17.2 flare event.

\begin{tabular}{lcccc}
\hline Index & $\begin{array}{c}\text { Meas. ave. } \\
\text { index } I_{\mathrm{M}}\end{array}$ & $\begin{array}{c}\text { Ratio } \\
\Delta \mathrm{EUV} / \Delta I_{\mathrm{M}}\end{array}$ & $\begin{array}{c}\text { Multipl. } \\
\text { factor } k\end{array}$ & $\begin{array}{c}\text { Pseudo } \\
\text { index } I_{\mathrm{P}}\end{array}$ \\
\hline$F_{10.7}$ & 274.4 & 0.51 & 1.28 & 348.5 \\
$F_{81 \mathrm{c}}$ & 140.5 & 0.95 & 2.38 & 334.4 \\
$S_{10.7}$ & 173.0 & 0.65 & 1.64 & 283.7 \\
$S_{81 \mathrm{c}}$ & 126.9 & 0.97 & 2.43 & 307.1 \\
$M_{10.7}$ & 175.6 & 0.67 & 1.69 & 296.8 \\
$M_{81 \mathrm{c}}$ & 132.6 & 0.96 & 2.41 & 319.6 \\
$Y_{10.7}$ & 182.2 & 0.49 & 1.24 & 225.9 \\
$Y_{81 \mathrm{c}}$ & 140.8 & 0.73 & 1.83 & 256.3 \\
\hline
\end{tabular}

In Tian et al. (2008b), the neutral density is given for EUV conditions during solar mean, $4 \mathrm{EUV}$ and $10 \mathrm{EUV}$. According to this, the neutral density during flare conditions (2.5 EUV) corresponding to the Sun at the age of $2.3 \mathrm{Gyr}$ can be estimated to be roughly $2.3 \times 10^{-11} \mathrm{~kg} \mathrm{~m}^{-3}$ for an altitude of about $500 \mathrm{~km}$. Compared to the observed and computed densities in Sect. 4, this estimate is more than one magnitude larger.

As far as the GRACE S/C is concerned, we have to mention that there was no perfect overlap between the satellite position and the flare impact location. A closer look to the S/C trajectory revealed that the satellite was behind the terminator when the flare irradiated the Earth's thermosphere at the dayside. As shown in Table 2, the $\mathrm{S} / \mathrm{C}$ has an orbit period of $94 \mathrm{~min}$, which is comparable with the flare exposure time of $\sim 90 \mathrm{~min}$ (see Sect. 2). Thus, it is likely that the satellite observed a weaker thermosphere response when it returned from Earth's shadow to the planet's dayside.

In the case of the JB08, it has to be emphasized that the model also uses 81-day average values of the solar indices to consider long-term activities. This causes inconsistencies if we compare the impact of a rather short flare irradiation with a permanent radiation of the same level, emitted by a Sun at the age of about $2.3 \mathrm{Gyr}$. In order to evaluate the JB08 model under such circumstances, we have to adapt the input values for the solar indices. Contrary to Sect. 4, where we discussed the impact of the flare event, we now assume a permanently high radiation. Thus, the constraint related to the lag time of the indices does no longer exist.

Based on TIMED/SEE measurements during the Halloween flare, we determined pseudo indices $I_{\mathrm{P}}$, which are intended to (1) reflect an analogue solar-like star at the age of approximately $2.3 \mathrm{Gyr}$ and (2) replace the actual averaged daily and 81-day solar indices $F_{10.7}, S_{10.7}, M_{10.7}$ and $Y_{10.7}$. Therefore, multiplication factors $k$ are calculated from the ratio $\Delta \mathrm{EUV} / \Delta I_{\mathrm{M}}$. This quotient is derived from a linear ap-

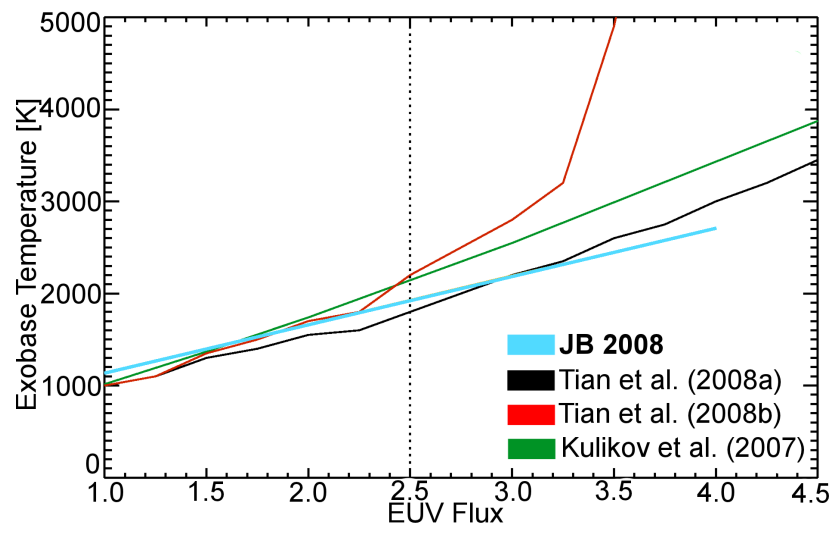

Fig. 8. Exobase temperatures obtained from the empirical thermosphere model JB08 and the theoretical models of Kulikov et al. (2007) and Tian et al. (2008a,b) under different solar EUV conditions. The vertical dotted line corresponds to the $2.5 \times$ EUV enhancement inferred from the X17.2 Halloween flare on 28 October 2003; if continuously emitted it would correspond to a $\sim 2.3 \mathrm{Gyr}$ old Sun.

proximation of the EUV flux measurements and the corresponding solar activity index ${ }^{3} I_{\mathrm{M}}$. This ratio is a measure of the increase of the specific solar index for a given increase of the EUV. In order to estimate the pseudo indices for the maximum of the flare $(2.5 \times \mathrm{EUV})$, we apply this ratio by:

$k=\frac{2.5 \times \Delta \mathrm{EUV}}{\Delta I_{\mathrm{M}}}$

which finally yield the pseudo indices $I_{\mathrm{P}}=k \cdot I_{\mathrm{M}}$. Table 4 summarizes the measured daily and 81-day averaged solar indices along with the determined ratio $\Delta \mathrm{EUV} / \Delta I_{\mathrm{M}}$ and the calculated multiplication factors $k$. The last column shows the resulting pseudo indices $I_{\mathrm{P}}$ related to the maximum of the X17.2 flare event.

A re-evaluation of the JB08 model with the pseudo indices yields the total mass density of $\sim 1.7 \times 10^{-11} \mathrm{~kg} \mathrm{~m}^{-3}$. This recalculated value now agrees better with the results from the theoretical models than the one previously obtained by using the averaged solar indices (Fig. 7, region 1). The result is between the findings from Kulikov et al. (2007) $\left(1.24 \times 10^{-11} \mathrm{~kg} \mathrm{~m}^{-3}\right.$ for $\left.3.0 \mathrm{EUV}\right)$ and the rough estimate of $\sim 2.3 \times 10^{-11} \mathrm{~kg} \mathrm{~m}^{-3}$ from the Tian et al. (2008a) model (2.5 EUV).

Based on this good agreement, we computed further indices for different solar EUV conditions. Since the JB08 model also provides exospheric temperatures, the following comparison is based on this quantity. Figure 8 illustrates the temperature at the exobase for increasing EUV fluxes, calculated with the empirical JB08 model and the results from the theoretical models (Kulikov et al., 2007; Tian et al., 2008a,b).

The theoretical models agree very well with the empirical JB08 model for the moderate present time solar EUV

\footnotetext{
${ }^{3} \mathrm{http}: / /$ sol.spacenvironment.net/ JB2008/indices.html
} 
conditions when the exobase is located at about $500 \mathrm{~km}$ with a temperature of $\sim 1000 \mathrm{~K}$. While the theoretical model of Kulikov et al. (2007) yields slightly higher exobase temperatures for the solar EUV flux larger than two times the present mean flux, the theoretical model of Tian et al. (2008a) agrees also quite well with the JB08 model at higher EUV fluxes. The highest exobase temperatures for solar EUV fluxes larger than 2.5 the present mean EUV flux result from the thermospheric model of Tian et al. (2008b) which includes an energetic electron transport model.

\section{Discussion and conclusions}

By analyzing the GRACE satellite drag data over eight years and comparing them with the empirical thermosphere models MSIS00 and JB08, our study indicates that the JB08 model reproduces the total mass density significantly better than the MSIS00 model - independent of the solar activity levels.

We investigated the effect of the EUV radiation during an extreme X17.2 flare event on the Earth's thermosphere. That flare was a more or less isolated phenomenon which could be separated from the CME-related particle events that occurred a day after. From the analysis of the peak EUV increase in the X17.2 flare, we found that both empirical models smooth out the related density and temperature enhancements in the thermosphere compared with the observed data. This is because the empirical models use daily average activity indices, while the flare lasted for only $\sim 90 \mathrm{~min}$. Furthermore, the GRACE satellite was at the nightside of the planet when the flare occurred. What the satellite did observe when it returned to the dayside was an aftermath of the flare impact on the thermosphere. We suppose that the GRACE S/C would have recorded a notably stronger density increase if it were on the dayside at the time when the flare occurred. In that case the actual difference between the observed density and the values calculated from the empirical models would be even larger than those in Fig. 7.

We compared the observed EUV flux during the flare peak and its related spectra with those of the solar analogue stars from the "Sun in Time" project (e.g. Ribas et al., 2005) and found that the peak EUV flux agrees with the EUV flux of a hypothetical Sun-like star or of the Sun at the age of about 2.3 Gyr. This corresponds to an EUV flux enhancement factor of approximately 2.5 times compared to that of the present Sun.

Based on the assumption that the Sun permanently emitted an EUV flux of this magnitude, we calculated pseudo solar activity indices for the JB08 model. A re-evaluation yielded an average exobase temperature of $\sim 1950 \mathrm{~K}$. This value agrees well with the exobase temperatures from the theoretical models (Kulikov et al., 2007; Tian et al., 2008a,b) if they employ similar EUV flux values. Thus, the agreement between the theoretical models and the empirical JB08 model validates the results for EUV fluxes $\leq 2.5$ times the present
EUV solar flux. Hence, if we assume an atmospheric composition similar to that of the present Earth, our study verifies the results from Tian et al. (2008a, p. 14, Fig. 8a, c) that the exosphere of the $\sim 2.3 \mathrm{Gyr}$ old Earth was approximately twice as hot $(\sim 2000 \mathrm{~K})$ as today and the exobase expanded to an altitude of $\sim 1000 \mathrm{~km}$, which is about two times higher than today.

Extrapolating the JB08 model to higher EUV fluxes which are expected to be emitted by the early Sun of approximately one Gyr age (2.5 to 4 times the present EUV flux), the results agree best with the model by Tian et al. (2008a). The difference between the various models concerning the exobase temperatures at EUV fluxes higher than approximately three times than today characterizes the current level of uncertainties inherent in the models. This is due to the insufficient understanding of the main processes that determine the energy balance of an $\mathrm{N}_{2}$-rich terrestrial thermosphere exposed to extremely high EUV radiation of the early Sun.

Furthermore, it is interesting that our analysis shows that CMEs may cause stronger density enhancements than the studied EUV radiation effect on the thermosphere triggered by the flare (see Fig. 5). Depending on the EUV flux enhancement, our finding indicates that, apart from the EUV radiation, energetic particle events may have even a stronger impact for the evolution of the early solar system planets or terrestrial exoplanets orbiting active young stars (Zaqarashvili et al., 2011; Khodachenko et al., 2007; Lammer et al., 2007). These effects could have a strong impact especially on Earth-like planets within orbits of close-in habitable zones around M-type stars.

From the results of our and former studies (e.g. Leblanc et al., 2002; Crider et al., 2005; Espley et al., 2005; Futaana et al., 2008), it becomes evident that extreme solar events can strongly affect the solar energy deposition in the upper atmosphere of a terrestrial planet. Such events alter the thermal structure, density, chemical composition and strongly enhance ion and neutral loss rates, which in turn are crucial factors in determining the planetary atmosphere evolution. Thus, we suggest that the combined in-situ experimental and theoretical studies of the solar plasma interaction can substantially advance our understanding of the principle driving mechanisms for the atmospheric loss processes, and have implications for the terrestrial solar and extrasolar planets' evolution pathways over geologic time scales.

\section{Appendix A}

When calculating the drag force coefficient $C_{\mathrm{F}}$, the interaction between the S/C surface and the molecules in the ambient atmosphere plays an important role. The coefficient is highly complex and can be split into two parts, namely the drag coefficient $C_{\mathrm{D}}$ and the lift force coefficient $C_{\mathrm{L}}$. The effective direction of drag coincides with the direction of the 


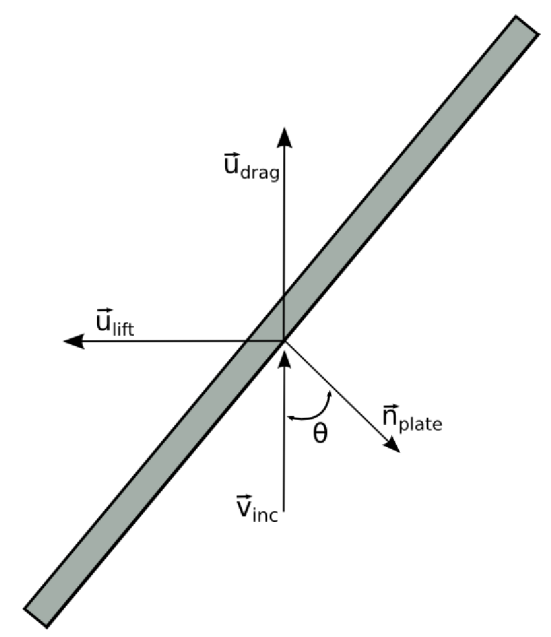

Fig. A1. Relation of the incident gas flow $\left(\boldsymbol{v}_{\text {inc }}\right)$, the plate normal $\left(\boldsymbol{n}_{\mathrm{i}}\right)$, and the drag $\left(\boldsymbol{u}_{\mathrm{drag}}\right)$ and lift $\left(\boldsymbol{u}_{\text {lift }}\right)$ unit vectors.

incident gas flow $\boldsymbol{v}_{\text {inc }}$. In contrast, the lift force $\boldsymbol{u}_{\text {lift }}$ acts perpendicular to it, in the plane spanned by the plate normal $\boldsymbol{n}_{\text {plate }}$ and the drag direction $\boldsymbol{u}_{\text {drag }}$ (Fig. A1).

Fundamental studies concerning the drag force coefficient were published in the late 1950s and early 1960s by, e.g., Sentman (1961) and Cook (1965). Without the knowledge of gas-surface interactions from satellites, Cook made a quite good and widely used estimate for the drag coefficient of $C_{\mathrm{D}}=2.2$. Modern technologies provide a better understanding for determining this force coefficient. The formalism underlying this study is an analytical expression of Sentman's treatise of the drag coefficient elaborated from Moe and Moe (2005).

For LEO satellites like GRACE, the average mean free path of the molecules near the exobase is almost $1 \mathrm{~km}$. Hence, the determination of aerodynamic forces and torques acting on the S/C are based on free-molecular flow theory (Storch, 2003). This means that there is no interference between the incident gas particles and the re-emitted molecules. Only the interaction with the surface molecules is of relevance. Assuming a free-molecule regime, the $\mathrm{S} / \mathrm{C}$ does not disturb the flow. Thus, it is permitted to split the complex shape of the satellite into simple flat plates and sum up the contribution from each of those (Sentman, 1961). Because Sentman's formalism also takes the effect of random thermal motion of the neutral atmosphere molecules into account, satellite panels that are nearly parallel to the flow are significant when computing the force coefficient.

At an altitude of $500 \mathrm{~km}$ the surface of a S/C gets contaminated with absorbed atomic oxygen molecules (e.g. Hedin et al., 1973; Moe and Moe, 1969, 2005) which is why incident molecules lose a large part of their kinetic energy. The re-emission should therefore be assumed to be almost completely diffuse. The velocity of re-emitted particles follows a Maxwell-Boltzmann distribution, in which the most prob- able velocity of the molecules $v_{\text {re }}$ can than be calculated as:

$v_{\text {re }}=v_{\text {inc }} \sqrt{\frac{2}{3}\left[1+\alpha\left(\frac{3 R T_{\mathrm{w}}}{v_{\text {inc }}^{2}}-1\right)\right]}$.

Here, $R$ represents the universal gas constant ( $8314.30 \mathrm{~J} \mathrm{kmol}^{-1} \mathrm{~K}^{-1}$ ) and $T_{\mathrm{w}}$ the surface temperature of the satellite panel which is assumed to be $273 \mathrm{~K}$ following Doornbos et al. (2009). The accommodation coefficient $\alpha$ indicates how closely the kinetic energy of the incoming molecule has adjusted to the thermal energy of the surface (Moe and Moe, 2005). For the present density determination process, we followed the recommendation of Koppenwallner (2008), who used $\alpha=0.90$ for his numerical examples.

Based on all these considerations, the drag and lift force coefficient are calculated following Moe and Moe (2005):

$C_{\mathrm{Di}}=\frac{A_{\mathrm{i}}}{A_{\text {ref }, \mathrm{i}}}\left[\frac{P_{\mathrm{i}}}{\sqrt{\pi}}+\gamma_{\mathrm{i}} Q Z_{\mathrm{i}}+\frac{\gamma_{\mathrm{i}} v_{\mathrm{re}}}{2 v_{\text {inc }}}\left(\gamma_{\mathrm{i}} \sqrt{\pi} Z_{\mathrm{i}}+P_{\mathrm{i}}\right)\right]$

and

$C_{\mathrm{Li}}=\frac{A_{\mathrm{i}}}{A_{\text {ref }, \mathrm{i}}}\left[l_{\mathrm{i}} G Z_{\mathrm{i}}+\frac{l_{\mathrm{i}} V_{\mathrm{re}}}{2 V_{\text {inc }}}\left(\gamma_{\mathrm{i}} \sqrt{\pi} Z_{\mathrm{i}}+P_{\mathrm{i}}\right)\right]$,

where $\gamma_{\mathrm{i}}$ and $l_{\mathrm{i}}$ denote $\cos (\theta)$ and $\sin (\theta)$ respectively, with $\theta$ being the angle between the incident gas particles and the specific plate normal. The auxiliary quantities $G, P, Q$ and $Z$ are functions of $\gamma$ and the molecular speed ratio $s . A_{\mathrm{i}} / A_{\text {ref,i }}$ describes the ratio between the true and the projected plate area. In this context one has to keep two things in mind: firstly, the influence of the co-rotating Earth atmosphere, and secondly, the deviation from the along track axis of the S/C, with respect to the orbital plane. The sum of the two factors leads to an angle of incidence of up to \pm 4 degrees depending on the course angle of the satellite. The calculation of the molecular speed ratio $s\left(s=v_{\text {inc }} / \sqrt{2 R T_{\mathrm{A}}}\right)$ requires temperatures of the ambient atmosphere $T_{\mathrm{A}}$. For the present study these temperature values are taken from the empirical thermosphere model Jacchia-Bowman 2008 (Bowman et al., 2008).

Since Shematovich et al. (2011) showed that photochemically produced hot oxygen atoms barely influence the satellite drag at altitudes below $500 \mathrm{~km}$, we neglected the contribution from the corona. The resulting variable drag force coefficient varies between 2.8 and 3.5 and thus yields approximately $60 \%$ lower neutral density values compared to a constant coefficient of $C_{\mathrm{D}}=2.2$ as proposed by Cook (1965).

Acknowledgements. This research has been supported by the Helmholtz Association through the research alliance "Planetary Evolution and Life" and through the joined Russian-Austrian project under the RFBR grant 09-02-91002-ANF-a and the Austrian Science Fund (FWF) grant I 199-N16. 
V. I. S. was partially supported by RFBR grant 08-02-00263. Additionally, the work was supported by the European FP7-PEOPLE2010-IRSES-269299 project - SOLSPANET.

H. L., W. H., H. I. M. L., and M. L. K. acknowledge the support by the FWF NFN project S116 "Pathways to Habitability: From Disks to Active Stars, Planets to Life", and the FWF NFN subprojects, S116 604-N16, S116606-N16, S116607-N16.

The authors thank Sean Bruinsma for his support concerning the GRACE accelerometer calibration and acknowledge the support from the FFG FEMtech - Project 836155 (Weltraumwetter).

Topical Editor C. Jacobi thanks three anonymous referees for their help in evaluating this paper.

\section{References}

Bettadpur, S.: GRACE 327-720 (CSR-GR-03-02) Gravity Recovery and Climate Experiment Product Specification Document, Technical Report, Center of Space Research, 2007.

Bowman, B. R., Tobiska, W. K., Marcos, F. A., Huang, C. Y., Lin, C. S., and Burke, W. J.: A New Empirical Thermospheric Density Model JB2008 Using New Solar and Geomagnetic Indices, AIAA/AAS Astrodynamics Specialist Conference, AIAA 20086438, 2008b.

Bruinsma, S., Forbes, J. M., Nerem, R. S., and Zhang, X.: Thermosphere density response to the 20-21 November 2003 solar and geomagnetic storm from CHAMP and GRACE accelerometer data, J. Geophys. Res., 111, 1-14, 2006.

Bruinsma, S. and Forbes, J. M.: Global observation of traveling atmospheric disturbances (TADs) in the thermosphere, Geophys. Res. Lett., 34, L14103, doi:10.1029/2007GL030243, 2007.

Bruinsma, S. and Forbes, J. M.: Properties of traveling atmospheric disturbances (TADs) inferred from CHAMP accelerometer observations, Adv. Space Res., 3, 369-376, 2008.

Bruinsma, S., Biancale, R., and Perosanz, F.: Calibration parameters of the CHAMP and GRACE accelerometers. IUGG XXIV General Assembly, Perugia, 2007.

Cook, G.: Satellite drag coefficients, Planet. Space Sci., 13, 929946, 1965

Crider, D. H., Espley, J., Brain, D. A., Mitchell, D. L., Connerney, J. E. P., and Acuña, M. H.: Mars Global Surveyor observations of the Halloween 2003 solar superstorm's encounter with Mars, J. Geophys. Res., 110, A09S21, doi:10.1029/2004JA010881, 2005.

Doornbos, E., Förster, M., Fritsche, B., Helleputte, T. V., Ijssel, J. V. D., Koppenwallner, W., Lühr, H., Rees, D., and Visser, P.: Air density models derived from multi-satellite drag observations, Technical Report, DEOS TU Delft, 317, 2009.

Dorren, J. D. and Guinan, E. F.: HD 129333: The Sun in its infany, Astrophysical Journal, 428, 805-818, 1994.

Encrenaz, T.: The Formation of Planets, Solar and ExtraSolar Planetary Systems, Springer Berlin/Heidelberg, 76-90, doi:10.1007/3-540-44807-1_6, 2001.

Espley, J. R., Cloutier, P. A., Crider, D. H., Brain, D. A., and Acuña, M. H.: Low-frequency plasma oscillations at Mars during the October 2003 solar storm, J. Geophys. Res., 110, A09S33, doi:10.1029/2004JA010935, 2005.

Futaana, Y., Barabash, S., Yamauchi, M., McKenna-Lawlor, S., Lundin, R., Luhmann, J. G., Brain, D., Carlsson, E., Sauvaud, J. A., Winningham, J. D., Frahm, R. A., Wurz, P., Holmström , M., Gunell, H., Kallio, E., Baumjohann, W., Lammer, H., Shar- ber, J. R., Hsieh, K. C., Andersson, H., Grigoriev, A., Brinkfeldt, K., Nilsson, H., Asamura, K., Zhang, T. L., Coates, A. J., Linder, D. R., Kataria, D. O., Curtis, C. C., Sandel, B. R., Fedorov, A., Mazelle, C., Thocaven, J. J., Grande, M., Koskinen, H. E. J., Sales, T., Schmidt, W., Riihela, P., Kozyra, J., Krupp, N., Woch, J., Fränz, M., Dubinin, E., Orsini, S., CerulliIrelli, R., Mura, A., Milillo, A., Maggi, M., Roelof, E., Brandt, P., Szego, K., Scherrer, J., and Bochsler, P.: Mars Express and Venus Express multi-point observations of geoeffective solar flare events in December 2006, Planet. Space Sci., 56, 873-880, doi:10.1016/j.pss.2007.10.014, 2008.

Güdel, M.: The Sun in Time: Activity and Environment, Living Reviews in Solar Physics, 4, 1-137, 2007.

Güdel, M., Guinan, E. F., and Skinner, S. L.: The X-Ray Sun in Time: A Study of the Long-Term Evolution of Coronae of SolarType Stars, Astrophysical Journal, 483, 947-960, 1997.

Guinan, E. F., Ribas, I., and Harper, G. M.: Far-Ultraviolet Emissions of the Sun in Time: Probing Solar Magnetic Activity and Effects on Evolution of Paleoplanetary Atmospheres, Astrophysical Journal, 594, 561-572, 2003.

Hedin, A. E., Hinton, B. B., and Schmitt, G. A.: Role of Gas-Surface Interactions in the Reduction of Ogo 6 Neutral Particle Mass Spectrometer Data, J. Geophys. Res., 78, 4651-4668, 1973.

Jacchia, L. G.: Revised static models of the thermosphere and exosphere with empirical temperature profiles. Smithsonian Astrophysical Observatory Special Report 332, 1971

Khodachenko, M. L., Ribas, I., Lammer, H., Grießmeier, J.-M., Leitner, M., Selsis, F., Eiroa, C., Hanslmeier, A., Biernat, H. K., Farrugia, C. J., and Rucker, H. O.: Coronal mass ejection (CME) activity of low mass M Stars as an important factor for the habitability of terrestrial exoplanets. I. CME impact on expected magnetospheres of Earth-like exoplanets in close-in habitable zones, Astrobiol., 7, 167-184, 2007.

Koppenwallner, G.: Comment on Special Section: New Perspectives on the Satellite Drag Environments of Earth, Mars, and Venus, J. Spacecraft Rockets, 45, 1324-1327, 2008.

Kulikov, Y. N., Lammer, H., Lichtenegger, H. I. M., Penz, T., Breuer, D., Spohn, T., Lundin, R., and Biernat, H. K.: A Comparative Study of the Influence of the Active Young Sun on the Early Atmospheres of Earth, Venus and Mars, Space Sci. Rev., 129, 207-243, 2007.

Lammer, H., Lichtenegger, H. I. M., Kulikov, Yu. N., Grießmeier, J.-M., Terada, N., Erkaev, N. V., Biernat, H. K., Khodachenko, M. L., Ribas, I., Penz, T., and Selsis, F.: Coronal mass ejection (CME) activity of low mass $\mathrm{M}$ stars as an important factor for the habitability of terrestrial exoplanets. II. CME-induced ion pick up of Earth-like exoplanets in close-in habitable zones, Astrobiol., 7, 185-207, 2007.

Lammer, H., Bredehöft, J. H., Coustenis, A., Khodachenko, M. L., Kaltenegger, L., Grasset, O., Prieur, D., Raulin, F., Ehrenfreund, P., Yamauchi, M., Wahlund, J. E., Grießmeier, J. M., Stangl, G., Cockell, C. S., Kulikov, Y. N., Grenfell, J. L., and Rauer, H.: What makes a planet habitable?, Astron. Astrophys. Rev., 181249, doi:10.1007/s00159-009-0019-z, 2009.

Leblanc, F., Luhmann, J. G., Johnson, R. E., and Chassefiere, E.: Some expected impacts of a solar energetic particle event at Mars, J. Geophys. Res., 107, A5, doi:10.1029/2001JA900178, 2002 . 
Lichtenegger, H. I. M., Lammer, H., Grießmeier, J. N., Kulikov, Y. N., von Paris, P., Hausleitner, W., Krauss, S., and Rauer, H.: Aeronomical evidence for higher $\mathrm{CO} 2$ levels during Earth's Hadean epoch, Icarus, 210, 1-7, doi:10.1016/j.icarus.2010.06.042, 2010.

Moe, M. and Moe, K.: The roles of kinetic theory and gassurface interactions in measurements of upper-atmospheric density, Planet. Space Sci., 17, 917-922, 1969.

Moe, K. and Moe, M.: Gas-surface interactions and satellite drag coefficients, Planet. Space Sci., 53, 793-801, 2005.

Montenbruck, O. and Gill, E.: Satellite Orbits Model Methods Applications,Springer, 369, ISBN-10: 354067280X/ISBN-13: 9783540672807, 2001.

Picone, J. M., Hedin, A. E., Drob, D. P., and Aikin, A. C.: NRLMSISE-00 empirical model of the atmosphere: Statistical comparisons and scientific issues, J. Geophys. Res. - Space Physics, 107, A12, doi:10.1029/2002JA009430, 2002.

Ribas, I., Guinan, E. F., Güdel, M., and Audard, M.: Evolution of the Solar Activity over Time and Effects on Planetary Atmospheres. I. High-Energy Irradiances (1-1700 ̊), Astrophysical Journal, 622, 680-694, 2005.

Scalo, S., Kaltenegger, L., Segura, A., Fridlund, M., Ribas, I., Kulikov, Y. N., Grenfell, J. L., Rauer, H., Odert, P., Leitzinger, M., Selsis, F., Khodachenko, M. L., Eiroa, C., Kasting, J., and Lammer, H.: M Stars as Targets for Terrestrial Exoplanet Searches and Biosignature Detection, Astrobiology, 7, 85-166, doi:10.1089/ast.2006.0125, 2007.

Sentman, L.: Free molecule flow theory and its application to the determination of aerodynamic forces, Technical Report, 1961.

Shematovich, V. I., Bisikalo, D. V., Krauss, S., Hausleitner, W., and Lammer, H.: Influence of the Hot Oxygen Corona on the Satellite Drag in the Earth's Upper Atmosphere, Solar System Res., 45, 231-239, 2011.
Storch, J. A.: Aerodynamic Disturbances on Spacecraft in FreeMolecular Flow. Aerospace Report No. TR-2003(3397)-1, 70, 2002.

Sutton, E. K., Nerem, R. S., and Forbes, J. M.: Density and Winds in the Thermosphere Deduced from Accelerometer Data, J. Spacecraft Rockets, 44, 1210-1219, 2007.

Tapley, B. D., Bettadpur, S., Watkins, M., and Reigbar, C.: The Gravity Recovery and Climate Experiment: Mission Overview and Early Results, Geophys. Res. Lett., 31, 9, doi:10.1029/2004GL019920, 2004.

Tian, F., Kasting, J. F., Liu, H. L., and Roble, R. G.: Hydrodynamic planetary thermosphere model: 1 . Response of the Earth's thermosphere to extreme solar EUV conditions and the significance of adiabatic cooling, J. Geophys. Res. (Planets), 113, 05008, doi:10.1029/2007JE002946, 2008a.

Tian, F., Solomon, S. C., Qian, L., Lei, J., and Roble, R. G.: Hydrodynamic planetary thermosphere model: 2 . Coupling of an electron transport/energy deposition model, J. Geophys. Res. (Planets), 113, 07005, doi:10.1029/2007JE003043, $2008 \mathrm{~b}$.

Tobiska, W. K., Bowman, B. R., and Bouwer, S. D.: Solar and geomagnetic indices for the JB2008 thermosphere density model, COSPAR CIRA Draft, Chapter 4, 2008.

Wu, S.-C., Kruizinga, G., and Bertiger, W.: Algorithm Theoretical Basis Document for GRACE Level-1B Data Processing V1.2, Data Processing, 741, 53, 2006.

Zahnle, K. and Walker, J. C. G.: Evolution of solar ultraviolet luminosity, Rev. Geophys. Space Phys., 20, 280-292, 1982.

Zaqarashvili, T. V., Oliver, R., Ballester, J. L., Carbonell, M., Khodachenko, M. L., Lammer, H., Leitzinger, M., and Odert, P.: Rossby waves and polar spots in rapidly rotating stars: implications for stellar wind evolution, A\&A, 532, A139, doi:10.1051/0004-6361/201117122, 2011. 